\title{
A Robust and Compact Recorder System for Ocean Bottom Seismometers
}

\section{INTRODUCTION}

The wide technological progress in marine sciences in the last ten years allows a better profit of natural resources, and risk and hazards prevention in the marine environment. Thus, the more developed countries have invested in technological innovation [1], which allows continuous sampling and supervision of different events to a regional scale. The autonomy of the equipment is a serious problem. At present it can run up to $72 \mathrm{~h}$ of continuous recording. Our main objective is to build an acquisition system which increases the recording time as well as the instrument size. Another objective in the new design is obtaining a light-weight system. This should allow the equipment to be installed in the desired zone without the help of an oceanographic vessel (and thus without its high costs). Obtaining a small-volume lightweight device is an important advantage compared to the existing seismometers [2], since it can be installed using one of the many fishing ships on the Mediterranean coasts.

The mini VC_OBS (VilanovaCarrack Ocean Bottom

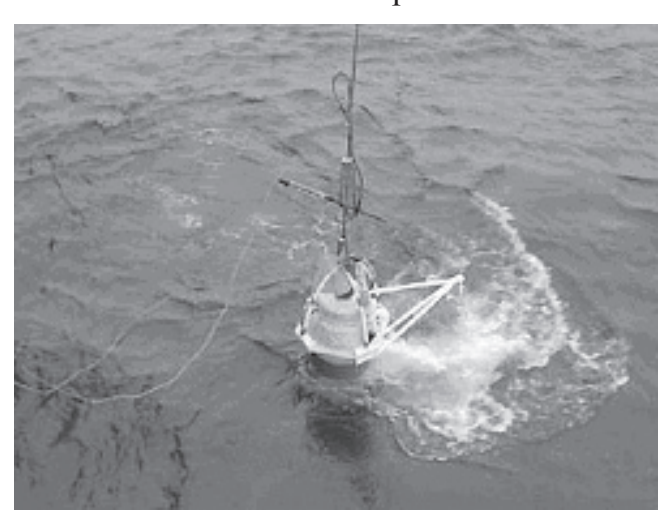

Fig 1.VC_OBS instrument layout

\section{II.ACQUISITIONSYSTEM}

The recorder system is based on a 24 -bit $\delta / \sigma$ ADC analog/digital converter. It takes the pre-amplified data from the hydrophone and the geophone and converts them to digital. It has 4 channels, one for the hydrophone and the other 3 for the geophone (1 vertical component plus 2 horizontal components). The sampling interval can be 128 or 256 samples per second, and the theoretical dynamic range is $144 \mathrm{~dB}$, with a signal to noise ratio of $130 \mathrm{~dB}$. The digital data arrives to a 32-bit M68332 Motorola processor from the ADC converter through a QSPI serial port and a memory card (Compact Flash 1GB) is used where the processed data is stored before it is downloaded to the hard disk. The use of the Flash Memory reduces the consumption of the complete system considerably because activating any mechanical element (hard disk's motor) uses a great deal of energy. The time needed to fill the card depends on the number of active channels and the width of the signal. In our experiments, it is usually filled within 24 hours. We can also use SCSI disks, each one with a capacity of $2.1 \mathrm{~GB}$. The data is stored in a compressed format which can be later visualized with the program DATADISP.EXE [14], and the stored data can be transferred to another storage media through a SCSI card.

To minimize energy consumption, the acquisition system only powers up when performing a data capture, activating the system through a combination of the pins PCS3, PCS2, PCS1 and PCS0 (Peripheral Chip Select) of QSPI bus. Then the board powers up and so all the logic chips. The uP reads multiplexed data and to do that it has to send a frame with channel address which is then stored to identify the data coming from each A/D converter. In each Read operation the converter sends a 32 bit frame to the uP. This frame contains information of the channel number, data and also the data stored in the status register of the converter. After the data has been received, time marks are added to them so we know exactly when they have been captured. This is done by software and the year, hour, minutes and seconds are added to every frame received. In general all A/D converters need a certain time to convert data 
(conversion time) and any communication attempt with the converter won't have the desired effects. Using the QSPI bus we can program the delays between transfers and the data size to acquire real data.

\section{III.DELTA-SIGMA A/D CONVERSION}

The sigma delta conversion has been in existence for many years, but recent technological advances now make the devices practical and their use is widespread. The converters have found homes in many applications such as communication systems, consumer and professional audio and geophysics measurements. The key feature of these converters is that they are the only low cost conversion method which provides both dynamic range and flexibility in converting low bandwidth input signals.

The need of having a decent $\mathrm{S} / \mathrm{N}$ and therefore high resolution persuaded us to choose an A/D converter with a high order modulator. The equation for the output of the modulator is given by: $\mathrm{y}_{\mathrm{i}}=\mathrm{x}_{\mathrm{i}-1}+\left(\mathrm{e}_{\mathrm{i}}-\mathrm{e}_{\mathrm{i}-1}\right)$ where e is the quantization noise.

Assuming the input signal is active enough to treat the error as white noise, the spectral density of the noise $\left(n=e_{i}-e_{i-1}\right)$ Can be expressed as:

$$
N(f)=E(f)\left|1-e^{(-j w) / f_{s}}\right|=2 e_{R M S}\left(\frac{2}{f_{s}}\right)^{\frac{1}{2}} \sin \left(\frac{w}{2 f_{s}}\right)(2)
$$

where $\left(\mathrm{V} / \mathrm{Hz}^{1 / 2}\right)$ is the units, $\mathrm{n}_{0}$ is the in-band quantization noise and $\mathrm{f}_{\mathrm{s}}$ is the sampling frequency.

The noise power in the bandwidth of interest is:

$$
n_{0}=e_{R M S} \frac{\pi}{\sqrt{3}}\left(\frac{2 f_{0}}{f_{s}}\right)^{\frac{3}{2}}
$$

This means that increasing the sampling frequency $f$ (which by default increases the oversampling ratio f / $\mathrm{f}_{0}$ or OSR) by a factor 2 will decrease the band noise by $9 \mathrm{~dB}$. In fact the generalized formula for the noise of an Mth order modulator is

$$
n_{0}=e_{R M S} \frac{\pi^{M}}{\sqrt{2 M+1}}\left(\frac{2 f_{0}}{f_{s}}\right)^{M+\frac{1}{2}}
$$

thus doubling the sampling frequency will decrease the in band quantization noise by $3(2 \mathrm{M}+1) \mathrm{dB}$. Figure 2 depicts the relationship between quantization noise, OSR and modulator order by showing the signal to noise ratio $(\mathrm{S} / \mathrm{N})$ vs OSR for a first, second and third order modulator.

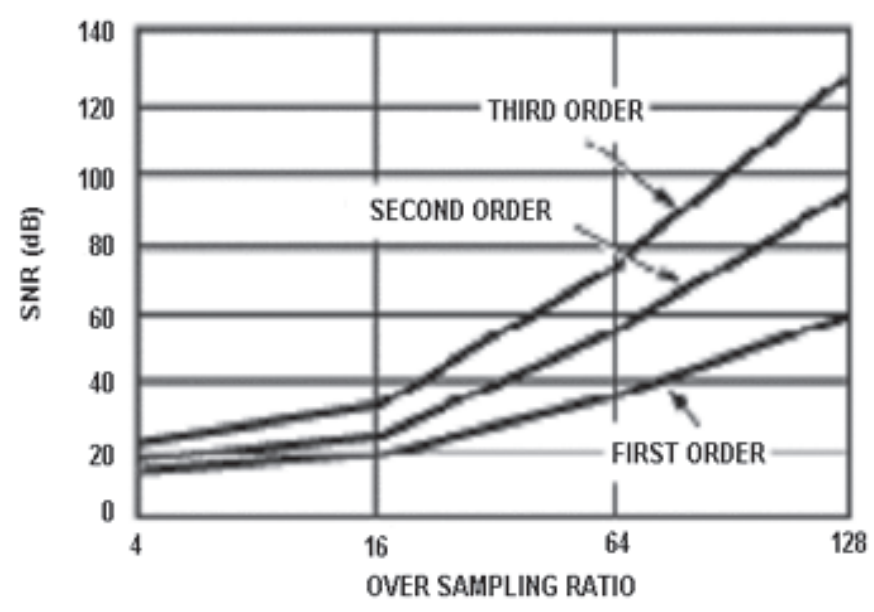

Fig 2: S/N vs Oversampling ratio for sigma delta modulators

The graph illustrates that as the OSR increases, the noise decreases ( $\mathrm{S} / \mathrm{N}$ increases) and that as the order of the modulator increases, the noise decreases.

The effects of oversampling on the noise is that the total quantization noise remains the same but the quantization noise in the bandwidth of interest is greatly reduced.

In particular we have used CS5321/22 Crystal A/D converters which are specially designed for seismic applications. They are intended for high resolution measurement signals below $1500 \mathrm{~Hz}$. They use delta sigma modulation for highly accurate conversions and they oversample $(256 \mathrm{kHz}$ or $128 \mathrm{kHz})$, virtually eliminating the need of external analog anti-alias filters. Figure 3 shows a simple block diagram of the recording system.

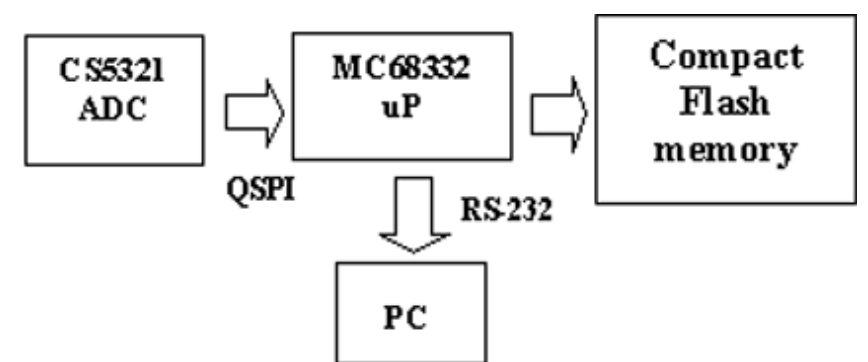

Fig. 3: Block diagram of the acquisition system

The modulator uses a fourth order Sigma Delta architecture whose $\mathrm{S} / \mathrm{N}$ is about $115 \mathrm{~dB}$. A FIR digital antialias filter supports the modulator eliminating the in-band noise.

\section{IV.DATAFORMAT}

C5321/22 Analog to digital converters have a 24 bit data format. The modulator outputs a ones density 
bit stream from its MDATA and $\overline{\text { MDATA }}$ pins proportional to the analog input signal, but at a bit rate determined by the modulator oversampling clock. For proper synchronization of the bit stream, the CS5320/21 has to be furnished with a synchronization signal. In our case, this signal is generated by hardware and its frequency is $256 \mathrm{~Hz}$.

As we said before, the communication protocol between the microcontroller and the $\mathrm{A} / \mathrm{D}$ board is under 32 bits data word. This includes the 24 bits A/D converter data plus 8 bit flags indicating the active channel number, data ready, synchronization and modulator ERROR.

This frame is then converted into another 32 bit format which contains the 24 bit data, (bits 0 to 23), 3 bits for filter pre-amp gain number ranging from 0 to 7 (bits 24 to 26). The 5 highest bits contain the minimum number of bits of a word which represents a data format including the sign information (bits 27 to 31$)$.

The gain bits are calculated using a software FIR digital filter and are considered as noise data bits. Then these bits are removed from the data. The same digital filter calculates the offset introduced by the modulator to the data and is then subtracted from the data. The following figure shows the 32 bits data formats:

\begin{tabular}{|l|l|l|}
\hline Wordlergth & Gain & Data \\
& & \\
\hline
\end{tabular}

These 32 bits data frames are saved in a data ring buffer. The buffer used here is not of a conventional first-in-first-out nature, but are time encoded so the position of the data within the buffer directly corresponds to one particular sampling instance. The organization of the buffer is such that there is a simple ring buffer of flags and pointers that map into a memory area containing the actual buffers of data. Figure 5 shows the structure of the ring buffer and actual data buffers.

At this point, data is read every second from the buffer and packed into a 64 bytes data packets with a variable word length in a way that in every packet, there is only data from one channel present. These data packets consist of 4 header bytes defined previously and 60 bytes of variable word length data. A compression algorithm calculates the largest data which will be included in the data packet and makes the rest of the data with the same word length. This way, in one data packet, all the data will have the same size (largest word length read from the data buffer).

The data packets have the following fields:

The header bytes include information on packet type, channel number, gain value and number data packets which fits inside a 60 bytes packet.

There are separate time mark packets for each data packet. These packets are 64 bytes long and contain information about packet type, number of active channels and synchronization flags. Both packets are stored in a Compact flash memory. The size of this memory is about $128 \mathrm{MB}$ although there are $1 \mathrm{~GB}$ cards already in the market.

\begin{tabular}{|l|l|}
\hline 4 header bytes & 60 bytes of Data \\
\hline
\end{tabular}

\section{V.RESULTS}

Tests have been carried out collecting data from the A/D board using a datalogger which integrates a 68332 microcontroller and a QSPI bus. A RS232 communication between datalogger and a lab PC has been implemented. After processing the results using the graphical programming language LabVIEW, the results have been satisfactory.

The data packets stored in Compactflash memory are monitored using the DATADISP.EXE [14] program.

The results shown are data packets collected in an experiment. Sampling rate is 256 samples/s. Time marks are shown at the beginning followed by data packets from each active channel. There are 4 active channels in the experiment and there are 10 data packets for each channel. The wordlength is (17 bits +1 sign bit $) /$ 
data except the last packet which can only fit 22 packets.

Another program called DOBSFILE.EXE [14] separates the data from each channel before visualization. Four files are generated containing data from each channel. In order to verify the data buffering and the compression algorithm, different input signals have been used to store compressed data onto the CompactFlash memory. Then data is recovered and decompressed using the above program. Then data is monitored using DATADISP.EXE [14] program.

More data is collected using the geophone as the input of the system using the same acquisition system. Figure 7 shows the results:

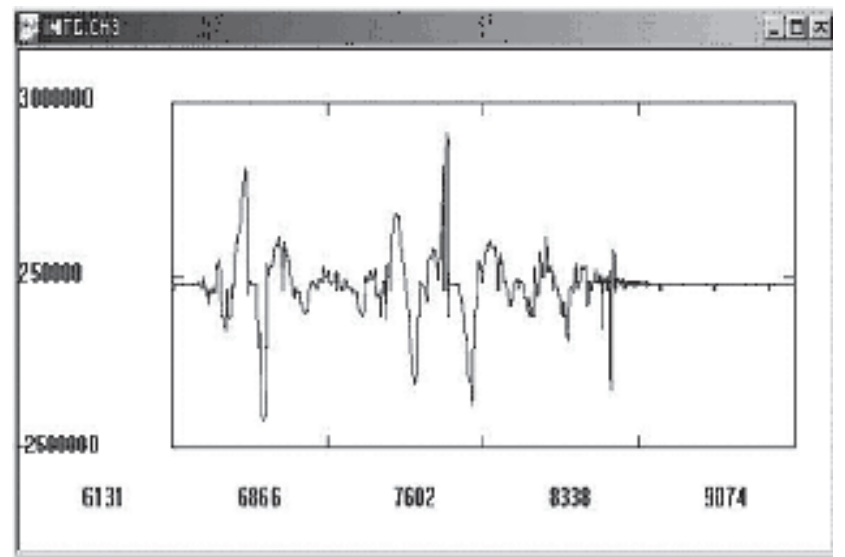

Fig 7: Data collected by a geophone in the lab

\section{CONCLUSIONS}

The main objective of this project is to increase the recording time of the instrument. This depends directly on the energy consumption of the system. The element which uses more energy is the SCSI hard disk.

More tests will have to be carried out on the instrument, but the final objective is to replace the SCSI hard disk with a bank of Compact flash memory cards. In this way we can further reduce the energy consumption of the instrument so the recording time will be increased a great deal.

From our first developed prototypes, we see the enormous possibilities of the OBS equipment in the technological innovation of marine environment (acoustic release, 24-bit A/D converters, interfaces and communication protocols, etc) and also in its applications. The OBS can be of particularly useful in civil engineering (stability, works in marine ports, and so on) and also in monitoring events of high and low frequency (microsismicity, tsunamis, etc). They are also important in the study of the subduction zones of high seismicity, and they allow the speed/deepness function of the sismogenic structures [4-5] to be obtained. They also allow mining prospecting and high definition of the cortical structure [6-12]

Regarding the scientific-technical technological innovation, it is important to point out the cumulated experience in the last years in the groups CSIC and UGBO-ICM-CSIC in the design of the first OBS. This refers to miniaturization equipment, easy handling and versatility, which allow the deployment of the OBS from any oceanographic vessel[13]. Our goals in the current and future prototypes are to improve the communications protocols, the efficiency in the energy distribution, the storage process and the easy access and system compatibility.

\section{REFERENCES}

[1] Prothero, W. A., A Digital Event-Recording Ocean Bottom Seismometer Capsule, Mar. Geophys. Res., 3, 119-141, 1976.

[2] A.Mànuel, J.Del Río, G.Olivar, H.Torruella , X. Roset, J.J.Dañobeitia, A. Bermúdez and T. Owen. New Generation of Ocean Bottom Seismometers. IEEE Instrumentation and Measurement $21-23$ May 2002

[3] Barash, T. W., C. G. Doll, J. A. Collins, G. H. Sutton and S. C. Solomon, Quantitative Evaluation of a Passively Leveled Ocean Bottom Seismometer, Mar Geophys. Res., 16, 347-363, 1994.

[4] Dañobeitia J.J., D. Cordoba, L.A. Delgado-Argote, F. Michaud, R. Bartolomé, M. Farran, R. Carbonell, F. Nuñez-Cornú, \& CORTES-P96 Working Group (1997). Expedition Gathers New Data on Crust Beneath the Mexican West Coast, EOS, vol . 78, 49, 565; 572.

[5] Flueh, E.R., Vidal, N., Ranero, C.R., Hojka, A., von Huene, R., J. Bialas, K. Hinz, D. Cordoba, Dañobeitia J.J. , and C. Zelt. (1998). Seismic Investigation of the Continental Margin off and onshore Valparaiso, Chile, Tectonophysics, 288, 251-263.

[6] Latraille, S, and L. M. Dorman, A standard format for the storage and exchange of natural and explosive source seismic data: the ROSE format, Mar. Geophys. Res., 6, 99-105, 1983.

[7] F. Michaud, J. J.Dañobeitia, R. Carbonell, R. Bartolomé, D. Córdoba, and L. Delgado-Argote. 2000. New insights about the oceanic crust entering the Middle American Trench off western Mexico $\left(17-19^{\circ} \mathrm{N}\right)$. Tectonophysics 318, Vol. 1-4, 187-200.

[8] JJ.Dañobeitia, A.Bermudez, J.Prades, P.Rodriguez, J.Díaz, T.Owen, A.Mànuel, X.Roset, G.Olivar, J Del Rio, E.Trullols. Nueva Generación de Sensores Submarinos Autónomos Ligeros SAAEI'01 Matanzas (Cuba) 17-19 Septiembre de 2001.

[9] Ye, S., Canales, J.P, Rihm, R., Dañobeitia, J.J. and Gallart, J., 1999, Crustal transect through the northern and northeastern part of the volcanic edifice of Gran Canaria, Jour. Geodyn., 28 (1), 3-26.

[10] Kuperman, W., W. Hodgkiss, LeRoy Dorman, K. Melville, G. Deane, G. D'Spain. Adaptive Beach Monitoring (Cruise Report) - off Camp Pendleton, CA, 1995

[11] Lewis, B.T.R, LeRoy M. Dorman, Recording Teleseisms on the Sea-floor; an Example from the Juan de Fuca Plate , Bull. Seismol. Soc. Am. February, 1998

[12] Canales, J.P., R.S. Detrick, S. Bazin, A.J. Harding, and J.A. Orcutt, Off-axis crustal thickness across and along the East Pacific Rise within the MELT area, Science, 280, 1218-1221, 1998 .

[13] Wooding, F.B, K.R Peal, J.T.Hallinan and J.A.Collins ORB-A new Ocean Bottom seismic data logger. Oceans 1998 MTS/IEEE Conference Proceedings, 28 Sept-1 Oct Nice (THF 4-2)

[14] T. Owen, Carrack Measurement Technology 